\title{
Preparation and characterization of polysaccharides/PVA blend nanofibrous membranes by electrospinning method
}

\author{
Carla Santos $^{\mathrm{a}, 1}$, Carla J. Silva ${ }^{\mathrm{a}, 1}$, Zsófia Büttel $^{\mathrm{b}}$, Rodrigo Guimarães ${ }^{\mathrm{b}}$, Sara B. Pereira ${ }^{\mathrm{b}}$, \\ Paula Tamagnini ${ }^{\mathrm{b}, \mathrm{c}}$, Andrea Zille ${ }^{\mathrm{b}, \mathrm{d}, *}$ \\ a Centro de Nanotecnologia e Materiais Técnicos, Funcionais e Inteligentes (CeNTI), 4760-034 Vila Nova de Famalicão, Portugal \\ b IBMC - Instituto de Biologia Molecular e Celular, Universidade do Porto, Porto, Portugal \\ ${ }^{\mathrm{c}}$ Faculdade de Ciências, Departamento de Biologia, Universidade do Porto, Porto, Portugal \\ d 2C2T - Centro de Ciência e Tecnologia Têxtil, Departamento de Engenharia Têxtil, Universidade do Minho, Guimarães, Portugal
}

\section{A R T I C L E I N F O}

\section{Article history:}

Received 9 May 2013

Received in revised form 29 August 2013

Accepted 4 September 2013

Available online $\mathrm{xxx}$

\section{Keywords:}

Polyvinyl alcohol (PVA)

Chitosan (CS)

Cyanobacteria

Extracellular polymeric substances (EPS)

Membrane

Electrospinning

Filtration

\begin{abstract}
A B S T R A C T
A series of polyvinyl alcohol (PVA), PVA/chitosan (CS) and PVA/cyanobacterial extracellular polymeric substances (EPS) blended nanofibrous membranes were produced by electrospinning using a microfiltration poly(vinylidene fluoride) (PVDF) basal membrane, for potential applications in water filtration. Nanofibres were obtained from solutions of $20 \%(\mathrm{w} / \mathrm{w})$ PVA with $1 \%(\mathrm{w} / \mathrm{w})$ CS or EPS, using a weight ratio of 60/40. Blended nanofibres have shown a smooth morphology, no beads formation and diameters between 50 and $130 \mathrm{~nm}$. Thermo-mechanical analysis demonstrated that there were inter and/or intramolecular hydrogen bonds between the molecules of PVA/CS and PVA/EPS in the blends. The electrospun blended PVA/EPS membrane showed better tensile mechanical properties when compared with PVA and PVA/CS, and resisted more against disintegration in the temperature range between 10 and $50^{\circ} \mathrm{C}$. Finally, the blended membranes have shown an increase in chromium binding capacity of $5 \%$. This is the first successful report of a blended membrane of electrospinned cyanobacterial polysaccharide with PVA.
\end{abstract}

\section{Introduction}

Since the mid 1990s, there has been a growing interest in the production of nanofibres by electrospinning. Electrospinning is a process carried out at room temperature that allows the production of polymer fibres with diameters in the sub-micron size range, through the application of an external electric field, keeping intact the bulk properties of the polymers. Electrospun membranes possess some unique structural features, such as a high surface to volume ratio and very good mechanical performance, properties that are determinant to their use in several applications such as air and liquid filtration, tissue engineering, optical and chemical

\footnotetext{
* Corresponding author at: 2C2T - Centro de Ciência e Tecnologia Têxtil, Departamento de Engenharia Têxtil, Universidade do Minho, Campus de Azurém, 4800-058 Guimarães, Portugal. Tel.: +351 226074900; fax: +351 226099157.

E-mail addresses: carla.correiasantos@gmail.com (C. Santos), CSilva@centi.pt (C.J. Silva), buttel.zsofi@hotmail.com (Z. Büttel), rodrigo.s.gui@gmail.com (R. Guimarães), sarap@ibmc.up.pt (S.B. Pereira), pmtamagn@ibmc.up.pt (P. Tamagnini), azille@det.uminho.pt (A. Zille)

1 These authors contributed equally to this work.
}

sensors (Liu, Wang, Ma, Hsiao, \& Chu, 2013). Although electrospun nanofibres have been used in industrial, consumer and defence filtration applications for more than twenty years, most of the research conducted to date has focused on synthetic polymers. Examples of electrospinning of natural polymers are limited to silk collagen, DNA, alginate, chitosan and more recently fibrinogen, gelatine and hyaluronic acid (Bhardwaj \& Kundu, 2010). Moreover, complex polysaccharides and proteins from bacteria and plant sources have not been sufficiently explored for their ability to be electrospun into nanofibres. Because of the very different chain conformations, hydrodynamic responses and repulsive forces in solution among the polyanions of natural polymers, the efficiency and reproducibility of the electrospinning process and the fibre uniformity remains a challenge, thus limiting their practical application (Schiffman \& Schauer, 2008). A method to overcome this drawback is blending the bio-polyelectrolytes with a non-toxic, water soluble, biocompatible, synthetic polymer such as Poly(vinyl alcohol) (PVA) since it can reduce repulsive forces within the charged biopolymer solutions and allow fibres spinning (Bonino et al., 2011). PVA is also inexpensive, chemically and thermally stable, and not easily degradable under most physiological conditions.

In this study, anionic and cationic biopolysaccharides blended with PVA were electrospinned into a polyvinylidene difluoride 
(PVDF) basal microfiltration membrane. The objective was to develop and characterize a mid-layer nanofibrous porous support for exploitable thin-film composite (TFC) membrane for water filtration. TFC membranes are asymmetric membranes with different layers consisting of different polymer materials that combine high flux with sufficient mechanical strength (Lau, Ismail, Misdan, \& Kassim, 2012). A high quality mid-layer porous support is the critical component for fabricating high-flux TFC membranes. The extremely high surface, bulk porosity and mechanical stability of the non-woven structures created by electrospinning overcomes the limitations of traditional membrane fabrication techniques and eases the application of an ultra-thin and defect-free selective barrier top layer for efficient air and liquid filtration applications (Tang et al., 2009). During the last years, several activities have been focused on the use of nanostructured biopolymers from renewable resources as alternatives to synthetic polymers for TFC membranes (Gunn \& Zhang, 2010). Among biopolymers, the polysaccharides are the most abundant and renewable natural polymers. Polysaccharides are predominantly neutral or acidic; however chitosan, the $\mathrm{N}$-deacetylated derivative of chitin, due to the presence of amino groups is a cationic polyelectrolyte $\left(\mathrm{p} K_{\mathrm{a}} \sim 6.5\right)$, one of the few occurring in nature. This gives chitin/chitosan singular chemical and biological characteristics such as biocompatibility, antibacterial properties, heavy metal ion chelation ability, gel-forming properties and hydrophilicity (Jia et al., 2007). Electrospun nanofibres based on chitosan have been investigated and various nanofibres products containing chitosan have been produced also for filtration purposes (Desai et al., 2009). Among the acidic polysaccharides and polysaccharide-based polymers, the cyanobacterial extracellular polymeric substances (EPS) possess unique characteristics that make them very attractive for biotechnological applications, including: (i) large number of different monosaccharides (usually 6-13), (ii) strong anionic nature due to the presence of two different uronic acids and sulphate groups, and (iii) hydrophobic behaviour conferred by the presence of esterlinked acetyl groups, peptidic moieties and deoxysugars (Mota et al., 2013; Pereira et al., 2009). The presence of the negatively charged uronic acid and sulfate groups has been associated to the anti-viral properties of cyanobacterial EPS (Kanekiyo, Hayashi, Takenaka, Lee, \& Hayashi, 2007), as well as its strong affinity towards metal ions (De Philippis, Colica, \& Micheletti, 2011; Pereira et al., 2011). In addition, the use of cyanobacterial EPS in an industrial context presents advantages compared to those polysaccharides obtained from algae and plants due to the higher growth rates of the cyanobacteria, the reproducible physicochemical properties of the EPS produced, and the overall economical costs. However, the information available on the rheological properties and biosynthetic pathways is still scarce, limiting their biotechnological applications (Pereira et al., 2009). In this work, the previously characterized released extracellular polymer of the marine cyanobacterium Cyanothece sp. CCY 0110 was used (Mota et al., 2013). Cyanothece's EPS are composed by 9 different monosaccharides (including two uronic acids, two pentoses and two deoxyhexoses), contain sulphate groups and peptides, and the polymer is remarkably thermostable and amorphous in nature (Mota et al., 2013).

The produced electrospinned PVA, PVA/chitosan and PVA/cyanobacterial EPS membranes were fully characterized. Atomic force microscopy (AFM), scanning electron microscopy (SEM), Energy-dispersive X-ray spectroscopy (EDS), dynamical and mechanical analysis (DMA), thermogravimetry (TGA), differential scanning calorimetry (DSC), and attenuated total reflection Fourier transform infrared spectroscopy (ATR-FTIR) analysis were performed to investigate the membranes morphology, diameter, structure, metal chelation efficiency, mechanical and thermal characteristics.

\section{Materials and methods}

\subsection{Materials}

PVA, 87-90\% hydrolyzed (wt. 30,000-70,000) was purchased from Sigma Aldrich (USA), Chitosan (deacetylation degree $84.8 \pm 1.2 \%$ ), ChitoClear hqg95-43,000, from Primex (Iceland), Polyvinylidene difluoride (PVDF) basal disc filter $(5 \mathrm{~cm}$ in diameter, porosity of $0.2 \mu \mathrm{m}$ ) from Sterlitech, USA. Cyanobacterial EPS were isolated from Cyanothece sp. CCY 0110 (Culture Collection of Yerseke, The Netherlands) cells grown in $10 \mathrm{~L}$ bioreactors (Clearboy ${ }^{\mathrm{TM}}$ transparent carboy with magnetic stirrer, Nalgene) with $7 \mathrm{~L}$ of ASNIII medium at $30^{\circ} \mathrm{C}$, under a continuous light regimen $\left(40 \mu \mathrm{E} \mathrm{m}^{-2} \mathrm{~s}^{-1}\right)$, with aeration $\left(5 \mathrm{~L} \mathrm{~min}^{-1}\right)$ and magnetic stirring (150 r.p.m.), according to the method described previously (Mota et al., 2013), precipitated with 2 volumes of $96 \%$ cold ethanol, collected with sterile metal forceps and lyophilized. Acetic acid was purchased from Pronolab and Dimethylsulfoxide from Riedel-de Haën. All other materials were purchased from Sigma-Aldrich and used without further purification.

\subsection{Electrospinning}

The electrospinning experiments were performed in a Nanon NF-103 (MECC, Japan) at room temperature, using a $10 \mathrm{ml}$ syringe with needles of $0.5 \mathrm{~mm}$ of inner diameter. An electric field that varied between 15 and $27 \mathrm{kV}$ was applied to all solutions, and the feed rate was varied from $0.1 \mathrm{ml} / \mathrm{h}$ to $1 \mathrm{ml} / \mathrm{h}$. The nanofibres produced were deposited on a collecting plate, on which the electric field was applied and the deposition distance of nanofibres to the collecting plate was controlled. Tests were performed with different solvents and with various polymers concentrations in order to determine the right amount of polymer to be used. Viscosity and conductivity of the examined solutions were measured in a viscometer (Fungilab Smart Series Rotational Viscometer) and in a conductivimeter (Thermo Scientific), respectively. The final membranes were obtained dissolving the PVA and EPS in distilled water (DW) at $80^{\circ} \mathrm{C}$ with constant stirring for at least $2 \mathrm{~h}$ and subsequently cooled to room temperature before electrospinning. As chitosan (CS) is soluble only in acidic medium, $1 \%(\mathrm{w} / \mathrm{w})$ acetic acid solution was used. The PVA solution (20\%, w/w) was mixed with CS and EPS solutions $(1 \%, w / w)$ in weight ratios PVA/CS and PVA/EPS of 60/40. Then, the mixed solutions were electrospinned onto a PVDF basal microfiltration disc filter. The electrospun membranes were further cross-linked by glutaraldehyde (GA) to maintain its morphology and prevent it from dissolution, using a procedure adapted from Wang et al. Electrospun membranes were immersed in a 5 mM GA and $0.01 \mathrm{~N} \mathrm{HCl}$ water solution for $6 \mathrm{~h}$, washed several times and kept in water before use (Wang et al., 2005).

\subsection{Scanning electron microscopy (SEM) and energy dispersive $X$-ray spectroscopy (EDS)}

Morphological analyses of nanofibres were carried out with an Ultra-high resolution Field Emission Gun Scanning Electron Microscopy (FEG-SEM), NOVA 200 Nano SEM, FEI Company. Secondary electron images were performed with an acceleration voltage at $5 \mathrm{kV}$. Backscattering Electron Images were realized with an acceleration voltage of $15 \mathrm{kV}$. Samples were covered with a film of $\mathrm{Au}-\mathrm{Pd}$ (80-20 weight \%) in a high-resolution sputter coater, 208HR Cressington Company, coupled to a MTM-20 Cressington High Resolution Thickness Controller. Atomic compositions of the membrane were examined with the energy dispersive spectroscopy (EDS) capability of the SEM equipment using an EDAX $\mathrm{Si}(\mathrm{Li})$ detector and an acceleration voltage of $5 \mathrm{kV}$. 


\subsection{Atomic force microscopy (AFM)}

AFM experiments were performed in a tapping mode in air using a Multimode AFM from Agilent, model 5500, at room temperature. Silicon cantilevers (AppNano) with a constant force between 25 and $75 \mathrm{~N} \mathrm{~m}^{-1}$ and a resonance frequency of $339 \mathrm{kHz}$ were used. The PicoView 1.10 software version was used for the simultaneous recording of the topography, phase and amplitude magnitudes of the images.

\subsection{Dynamic mechanical analysis (DMA)}

Dynamical and mechanical analysis was performed on a DMA 8000 (Perkin Elmer, USA) in tension mode according to an internal method based on standard ASTM D4065-01. The temperature dependence of the storage modulus and loss tangent was measured in the temperature range from -50 to $120^{\circ} \mathrm{C}$ at $2^{\circ} \mathrm{C} / \mathrm{min}$.

\subsection{Thermal gravimetric analysis (TGA)}

The thermogravimetric analysis was carried on a Pyris 1 TGA (Perkin Elmer, USA), according to the standard ISO 11358:1997(E). The TGA trace was obtained in the range $40-900^{\circ} \mathrm{C}$ under nitrogen atmosphere with a flow rate of $20 \mathrm{~mL} / \mathrm{min}$ at rise of $10^{\circ} \mathrm{C} / \mathrm{min}$. The samples were previously dried at $60^{\circ} \mathrm{C}$ for $1 \mathrm{~h}$ and placed into a porcelain sample pan. The graph was plotted with weight (percentage) vs. temperatures.

\subsection{Differential scanning calorimeter (DSC) analysis}

The DSC analysis was carried on a Power compensation Diamond DSC (Perkin Elmer, USA) with an Intracooler ILP, based on the standards ISO 11357-1:1997, ISO 11357-2:1999 and ISO 11357$3: 1999$. The samples were previously dried at $60^{\circ} \mathrm{C}$ for $1 \mathrm{~h}$ and placed into an aluminium sample pan. The analysis was carried out in nitrogen atmosphere with a flow rate of $20 \mathrm{~mL} / \mathrm{min}$ and heating rate of $10^{\circ} \mathrm{C} / \mathrm{min}$. The thermogram was obtained in the range of $-50-200^{\circ} \mathrm{C}$. This upper limit for DSC was selected for being the initial decomposition temperature for all the tested polymers as seen by TGA. The graph was plotted with heat flow vs. temperatures.

\subsection{Attenuated total reflectance-fourier transform infrared spectroscopy (ATR-FTIR)}

The chemical structure of the nanofibre mats was characterized using an attenuated total reflectance Fourier transform (ATR-FTIR) spectrophotometer (Perkin Elmer, Spectrum 100). Each spectrum was acquired in transmittance mode on a ZnSe ATR crystal cell by accumulation of 256 scans with a resolution of $4 \mathrm{~cm}^{-1}$ and a wave number range of $4000-600 \mathrm{~cm}^{-1}$.

\subsection{Metal chelation efficiency}

The synthesized membrane filters were tested by dead-end filtration of a standard solution of hexavalent chromium $(2 \mathrm{mg} / \mathrm{L})$ prepared using potassium dichromate $\left(\mathrm{K}_{2} \mathrm{Cr}_{2} \mathrm{O}_{7}\right)$. The set-up consisted of a $300 \mathrm{~mL}$ bench stainless steel tangential flow stirred cell (Sterlitech, HP4750) pressurized with air. The prototype filtration cell allows testing membranes with a $5 \mathrm{~cm}$ diameter and a working volume of $200 \mathrm{~mL}$. The solution in the chamber was stirred by a Teflon-coated magnetic bar at $300 \mathrm{rpm}$. The solution of hexavalent chromium ( $2 \mathrm{mg} / \mathrm{L})$ prepared using potassium dichromate $\left(\mathrm{K}_{2} \mathrm{Cr}_{2} \mathrm{O}\right.$ ) $)$ was circulated through the filter membrane five times. All experiments were carried out at $25^{\circ} \mathrm{C}$ using $50 \mathrm{~mL}$ metal solution and a pressure of at 3 bar. The concentration of $\mathrm{Cr}(\mathrm{VI})$ was calculated by an internal method from the absorbance at $542 \mathrm{~nm}$
Table 1

Conductivity and viscosity values of the polymer solutions tested for electrospinning (in bold is presented the concentration value of the solutions used for building the nanofibres mats)

\begin{tabular}{lcc}
\hline Polymer blend $\left(\mathrm{w} / \mathrm{w} \%\right.$ in $\left.\mathrm{H}_{2} \mathrm{O}\right)$ & Conductivity $\left(\mu \mathrm{Scm}^{-1}\right)$ & Viscosity $(\mathrm{cP})$ \\
\hline PVA & & \\
$\mathbf{1 2} \%$ PVA & $\mathbf{8 7 4} \pm \mathbf{9}$ & $\mathbf{9 6} \pm \mathbf{3}$ \\
$20 \%$ PVA & $1095 \pm 25$ & $1502 \pm 1$ \\
& & \\
PVA/EPS & & \\
$0.3 \%$ EPS + 6\% PVA & & $110 \pm 2$ \\
$0.3 \%$ EPS + 8\% PVA $^{\text {a }}$ & $784 \pm 10$ & $94 \pm 2$ \\
$0.1 \%$ EPS + 6\% PVA & $804 \pm 1$ & $23 \pm 3$ \\
$0.2 \%$ EPS + 12\% PVA & $948 \pm 6$ & $174 \pm 14$ \\
$\mathbf{0 . 5 \%}$ EPS + 12\% PVA & $\mathbf{1 1 4 9} \pm \mathbf{2 6}$ & $\mathbf{5 6 3} \pm \mathbf{3}$ \\
& & \\
PVA/CS & $\mathbf{1 2 7 4} \pm \mathbf{2 0}$ & $\mathbf{4 4 2} \pm \mathbf{1 2}$ \\
$\mathbf{0 . 5 \%}$ CS + 12\% PVA & $1602 \pm 11$ & $479 \pm 8$ \\
$0.7 \%$ CS + 9\% PVA & $1791 \pm 2$ & $284 \pm 14$ \\
$0.9 \%$ CS + 5\% PVA & $3050 \pm 14$ & $678 \pm 24$ \\
$1.6 \%$ CS + 4\% PVA & & \\
\hline
\end{tabular}

a Solvent 50/50 DMSO: $\mathrm{H}_{2} \mathrm{O}$.

using a UV-vis spectrophotometer (Perkin Elmer, Lambda35). Each experiment was performed in triplicate. The $t$-Student test was used to test the statistical significance of the attained results at $p<0.001$.

\section{Results and discussion}

\subsection{Electrospinning}

The shape and the size of nanofibres are governed by several parameters such as concentration, viscosity, conductivity, and surface tension of the polymer solution. Although they are all important parameters, polymer solution viscosity and conductivity are the main parameters that affect the final characteristics of electrospun fibres. Therefore, prior to electrospinning, the different polymeric systems used to produce electrospun mats were characterized and optimized in terms of the above properties. Due to its plasticizing properties PVA has an important role in the blending process, in the way that eases the uniformization and the bonding of the chitosan and exopolysaccharide solutions. So, the optimization for a PVA-based solution was the starting point of this study Different concentrations of aqueous PVA solutions were prepared and tested in the electrospinning equipment $(5,10,12$ and $20 \%$ $\mathrm{w} / \mathrm{w}$ of PVA). Only for two of these PVA solutions (12\% and $20 \%$ $(\mathrm{w} / \mathrm{w})$ - Table 1) it was possible to obtain nanofibres, due to their viscous and conductive properties. In fact, changes in polymer concentration resulted in modifications of the physical properties of the nanofibres. If the PVA concentration is too low the system will perform mere electrospraying, without suitable nanofibres formation, or it will develop large beads (polymer agglomerates) at higher PVA concentrations. This phenomenon is due to the increase of the solution surface tension, being also affected by the applied charge density and voltage (Jia et al., 2008). The increased viscosity beyond a critical value enabled the charged jet to totally withstand the columbic stretching force, resulting in uniform and smooth fibres. Using 20\% (w/w) PVA solutions, fibres with diameters ranging between 74 and $181 \mathrm{~nm}$ were attained (data not shown). However, when the $20 \%(\mathrm{w} / \mathrm{w})$ PVA solution was blended with the polysaccharides (CS and EPS) solutions it was impossible to obtain suitable electrospun conditions. It was also seen that solutions of pure EPS and CS were not electrospinnable, independently of their polysaccharide concentration. This is mainly due to the high surface tension and conductivities of chitosan acetic acid solutions and to the high viscosity and ionic strength of the negatively charged EPS solutions (Duan et al., 2006; Peresin, Habibi, Zoppe, Pawlak, \& Rojas, 2010). Electrospun nanofibres could not be generated from pure chitosan 
and EPS solutions, but could be fabricated from blended systems of polysaccharides and PVA. As shown in Table 1 different concentration of EPS, CS and PVA were tested for blended solutions. The purpose of this study was to reduce the percentage of PVA in solution to the minimum value that allows the fibre production and increasing the concentration of polysaccharides in order to produce nanofibers of these polymers. The voltage was also tested between 15 and $27 \mathrm{kV}$ without significant effects in fibres morphology and electrospinnability (data not shown). The results showed that the nanofibres synthesis is polymer-composition dependent, as expected. Increasing the polysaccharide concentration, the solution viscosity and conductivity increased. It is known that solution parameters as viscosity, electrical conductivity and surface tension affect directly the fibre dimensions and morphology. Viscosity is one of the most important solution parameters. Both solution viscosity and concentration are related by Berry Number (Be), defined by Eq. (1):

$\mathrm{Be}=[\eta] * C$

where $[\eta]$ is intrinsic polymer viscosity and $C$ is the polymer solution concentration. When $\mathrm{Be}<1$, polymer molecules in solution are sparsely distributed and there is a low probability of an individual molecule to bind with another. As a result, only beads and beaded fibres are formed. At $1 \leq \mathrm{Be} \leq 2.7$, entanglement probability increases and favourable conditions for fibre production take place. This Be number is convenient for nanofibre production and used in this work. At $B e \geq 2.7$, polymer chain entanglements probability increases and the average fibre diameter goes above the micrometre range (Leung \& Ko, 2011).

The electrical conductivity of solution also plays an important role on fibre morphology. Electrical conductivity is associated to the number of charges in solution, which causes instabilities in the electrospinning process. The addition of charge carriers such as salts or conductive filler particles have an influence on the conductivity of the solution and they may promote two phenomena having opposite effects on fibre formation and diameter: they may increase flow rate, which can lead to larger fibres or they may increase net charge density, which suppresses the Rayleigh instability and enhances the whipping instability, leading to the formation of bead free fibres and smaller fibre diameter (Heikkila \& Harlin, 2009).

Fig. 1 shows the SEM and AFM images of the PVA/polysaccharide blended electrospun fibres using $12 \%(\mathrm{w} / \mathrm{w})$ of PVA and $0.5 \%(\mathrm{w} / \mathrm{w})$ of EPS and CS. When the concentration of polysaccharides in the blend solutions was higher than $0.5 \%(\mathrm{w} / \mathrm{w})$, the electrospinning process was unstable due to the high viscosity and conductivity. The blended nanofibres have shown a uniform and smooth morphology with no beads formation and diameters between 60 and $130 \mathrm{~nm}$ for CS and between 50 and $120 \mathrm{~nm}$ for EPS (Fig. $1 \mathrm{~b}$ and c). The values attained for the nanofibres diameter were lower than the $20 \%(w / w)$ pure PVA nanofibres, due to the high surface charge densities of the polysaccharides electrospinning solutions, which induced more extensive filament stretching during jet whipping. When the same amount of PVA used in the blended nanofibres $(12 \%(\mathrm{w} / \mathrm{w}))$ was electrospinned alone, brittle and thin nanofibres with large number of beads were observed (Fig. 1a), due to lower conductivity and viscosity.

To confirm that the electrospun fibres contained the polysaccharides in their structure an EDS analysis was performed. The elemental composition in the electrospun fibres is shown in Table 2. PVA has only oxygen and carbon in its structure. However, chitosan contains nitrogen in its amine and acetylamine groups on its backbone and the cyanobacterial EPS used in this work contain sulphate groups. The PVA/chitosan membranes contained carbon, oxygen, and nitrogen. The nitrogen amount was $1.89 \mathrm{At} \%$, in close agreement with the theoretical nitrogen composition, indicating that PVA and chitosan were efficiently blended in the nanofibres. On
Table 2

Variation of weight and atomic percentages of the atoms C, O, N and S in the electrospun nanofibres.

\begin{tabular}{|c|c|c|c|c|c|c|}
\hline \multirow[t]{2}{*}{ Element } & \multicolumn{2}{|l|}{ PVA } & \multicolumn{2}{|l|}{ PVA/CS } & \multicolumn{2}{|c|}{ PVA/EPS } \\
\hline & Wt\% & At\% & Wt\% & At\% & Wt\% & At\% \\
\hline $\mathrm{C}$ & 44.30 & 51.44 & 42.27 & 49.26 & 37.32 & 44.25 \\
\hline $\mathrm{O}$ & 55.70 & 48.56 & 55.84 & 48.85 & 61.97 & 55.40 \\
\hline $\mathrm{N}$ & - & - & 1.89 & 1.89 & - & - \\
\hline S & - & - & - & - & 0.71 & 0.35 \\
\hline Total & 100.00 & 100.00 & 100.00 & 100.00 & 100.00 & 100.00 \\
\hline
\end{tabular}

the other hand, the PVA/EPS membrane analysis revealed the presence of sulphur in its structure. The relative low value $(0.35 \mathrm{At} \%)$ is due to the fact that sulphate groups in the heteropolymeric structure of the cyanobacterial EPS are not probably part of the polysaccharide-repeating units as for nitrogen in chitosan. An interesting fact is also the higher amount of oxygen (55 At\%) in the PVA/EPS nanofibres when compared with PVA and PVA/CS nanofibres (about 49 At\%). This could be attributed to the high amount of two different uronic acids (7\% expressed as molar \%) in cyanobacterial EPS (Mota et al., 2013). Sulphate groups and uronic acids contribute to the anionic nature of the EPS, conferring a negative charge and a 'sticky' behaviour to the overall macromolecule.

\subsection{Attenuated total reflectance-Fourier transform infrared spectroscopy (ATR-FTIR)}

The ATR-FTIR spectra (Fig. 2) of nanofibres mats of PVA, PVA/EPS and PVA/CS showed the dominant absorption peaks at $3320,2940,1430,1736,1090$ and $850 \mathrm{~cm}^{-1}$, which were respectively attributed to the $v(\mathrm{O}-\mathrm{H}), v \mathrm{~s}\left(\mathrm{CH}_{2}\right), \delta(\mathrm{CH}-\mathrm{O}-\mathrm{H}), v(\mathrm{C}=\mathrm{O})$, $v(\mathrm{C}-\mathrm{O})$ and $\mathrm{v}(\mathrm{C}-\mathrm{C})$ of pure PVA (Charernsriwilaiwat, Opanasopit, Rojanarata, Ngawhirunpat, \& Supaphol, 2010). The PVA/CS and PVA/EPS blended nanofibre mats exhibited in general the same peaks as those found in the PVA nanofibres mats due to the low amount of pure polysaccharide polymers (dashed lines) in PVA matrix. However, in the PVA/CS nanofibre mats, a small peak is visible at $1654 \mathrm{~cm}^{-1}$, belonging to the carbonyl stretching of the secondary amide band (amide I) of the pure chitosan (Yan et al., 2013). Moreover, the CS and PVA/EPS spectra exhibited a faint shoulder at $898 \mathrm{~cm}^{-1}$, characteristic of their saccharide structure. The intensities of the carboxylic band at $1568 \mathrm{~cm}^{-1}$ attributed to the acetate group of the PVA show a relative diminution. These data suggest that the reaction of the PVA with EPS and CS has indeed occurred by forming acetal bridges (Mansur, Sadahira, Souza, \& Mansur, 2008).

\subsection{Thermogravimetry (TGA) analysis}

TGA analysis of the polymers showed that the degradation takes place in three well-differentiated steps for EPS and PVA (Fig. 3a and c) and in two steps for chitosan (Fig. 3b). The first step for all the polymers is an initial weight loss due to moisture content between 35 and $100^{\circ} \mathrm{C}$. The higher initial moisture content in the EPS and CS samples is the result of an increased level of carboxyl groups in the polysaccharides. This is attributed to the direct positive relationship between the carboxyl contents and the affinity of the polysaccharide for interaction with water molecules (Kumar, Joo, Choi, Koo, \& Chang, 2004). In the EPS thermogravimetric analysis (Fig. 3a) the weight remains constant until a second phase attributed to polysaccharide decomposition that starts around $234 \pm 1{ }^{\circ} \mathrm{C}$ and continues until $340 \pm 1{ }^{\circ} \mathrm{C}$ with weight loss of $23.3 \pm 0.6 \%$ matching to the start of the degradation of one of its components. Slowly, a progressive carbonized structure was formed as the temperature increased further. The third phase of 

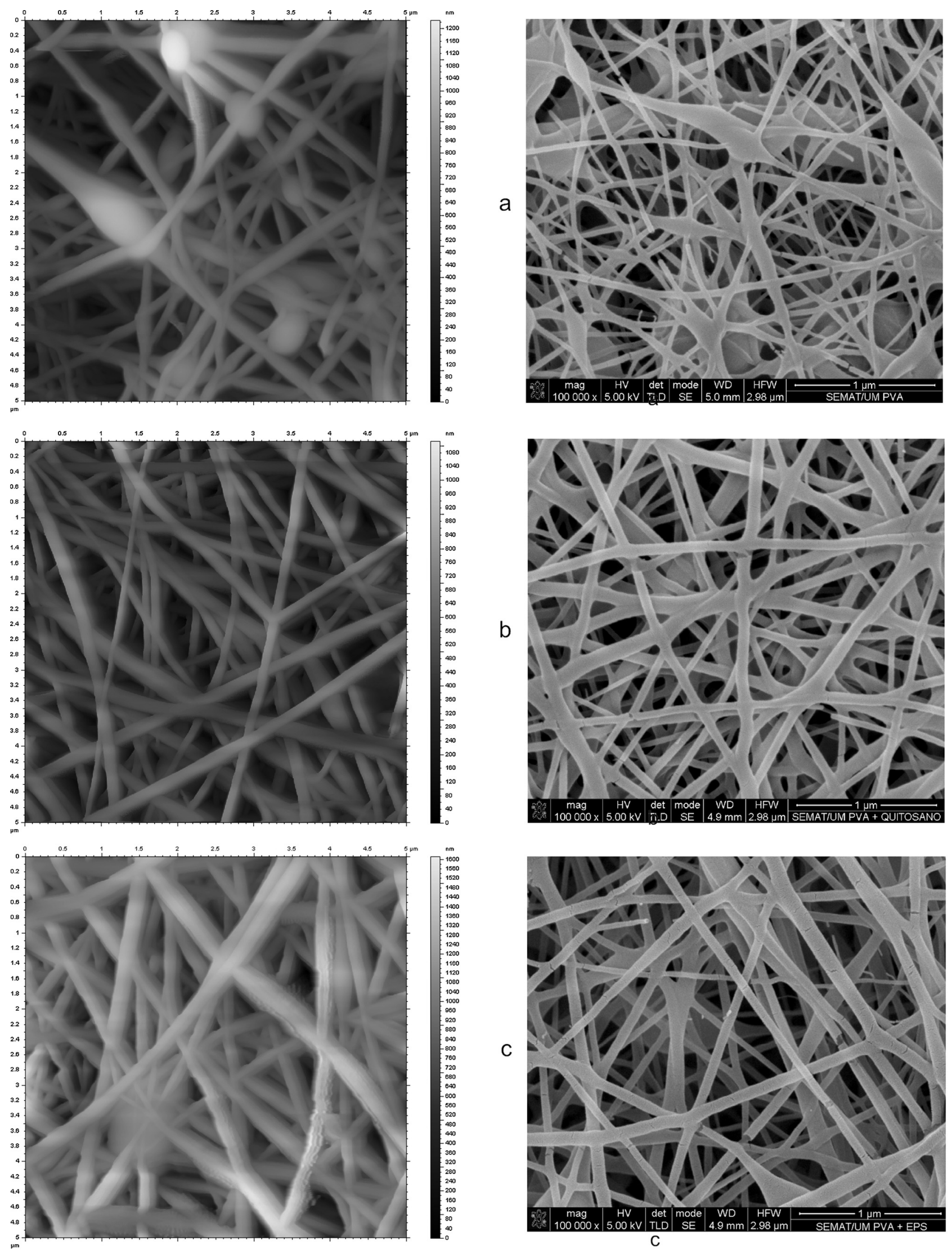

Fig. 1. AFM images, with an analysis area of $5 \times 5 \mu \mathrm{m}$, and SEM images of the membranes obtained from PVA (a), PVA and chitosan (b), and PVA and exopolysaccharides (c) solutions. 


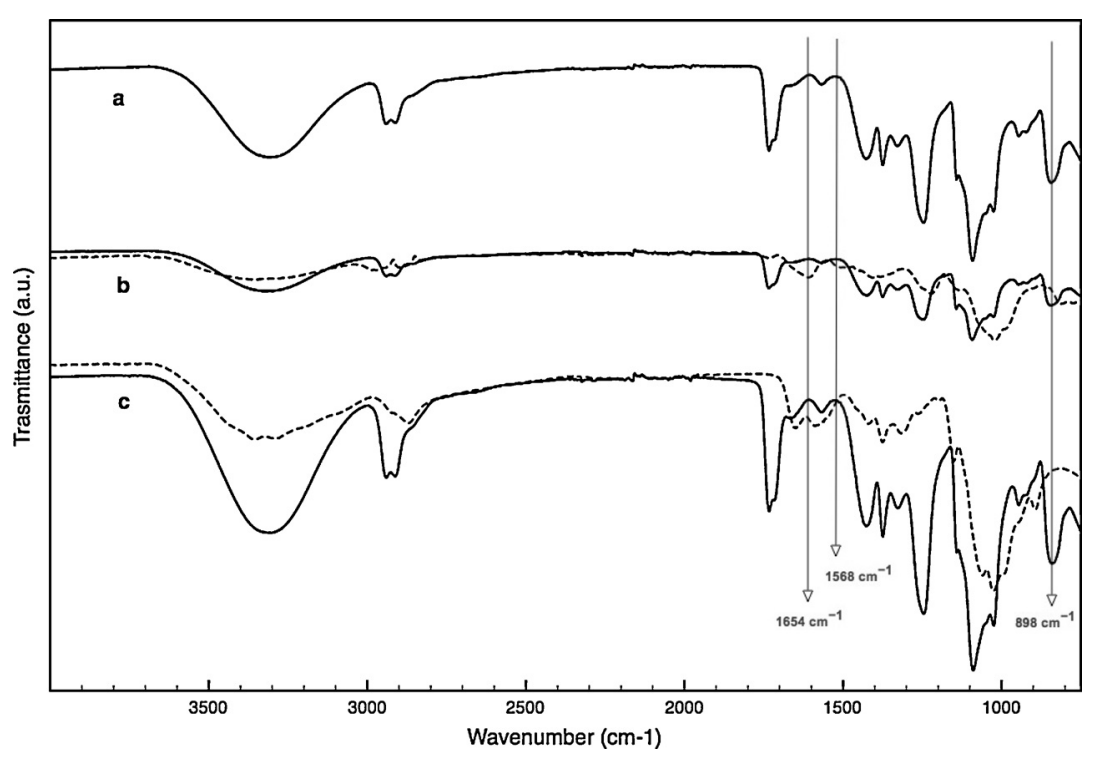

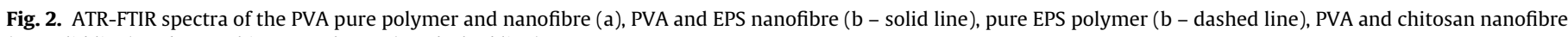
(c - solid line) and pure chitosan polymer (c - dashed line).

degradation from $769 \pm 8^{\circ} \mathrm{C}$ to $843 \pm 9{ }^{\circ} \mathrm{C}$, with an average weight loss of $25 \pm 2 \%$, represents the degradation of the remaining components on the sample. When the test ended at $900^{\circ} \mathrm{C}$, the sample had lost approximately half of the starting mass, showing a residual mass of about $45 \pm 2 \%$. This high thermostability, with the consequent high solid residue content, might be due to the complex and heterogeneous molecular structure of the EPS, including the presence of sulfate groups and uronic acids (Mota et al., 2013), and to the presence of cations $\left(\mathrm{Na}^{+}, \mathrm{K}^{+}, \mathrm{Ca}^{2+}\right)$, which acted like a bridge between the different charged sugar moieties. Two weight losses are observed in the chitosan TGA curve (Fig. 3b). The weight loss ( $\sim 3 \%)$ at $50-100^{\circ} \mathrm{C}$ is due to the moisture vaporization. The other weight loss ( $\sim 60 \%)$ starting at $291 \pm 2{ }^{\circ} \mathrm{C}$ is due to the degradation of chitosan molecule. At the end of the experiment at $900^{\circ} \mathrm{C}$, the sample of chitosan shows a residual mass of about $29.7 \pm 0.5 \%$ of the starting mass. These results are similar to previous studies of other chitosan-based polymers that have reported a decomposition temperature of about $300^{\circ} \mathrm{C}$ (Chen, Wang, Mao, Liao, \& Hsieh, 2008). The TGA of the PVA (Fig. 3c) exhibits three zones of mass loss, corresponding to the degradation of the polymer components in accordance with the reported literature (Mbhele et al., 2003). After the water loss step, the decomposition occurred mainly in the second degradation step that starts at $310 \pm 2{ }^{\circ} \mathrm{C}$ where it loses about $77 \%$ of its mass and reflects the decomposition of side PVA chain (Peng \& Kong, 2007). The second smaller step at $430^{\circ} \mathrm{C}$ is attributed to the PVA main chain degradation (Shao et al., 2003). At $900^{\circ} \mathrm{C}$ a residue of about $4.5 \%$ of the starting mass can be observed. Due to the low amount of polysaccharides in the blended nanofibres, no differences in TG degradation profile could be observed. The degradation profiles of the three types of nanofibres are very similar to each other (Fig. 3 - dot lines - d-f) fitting the degradation profile of PVA.

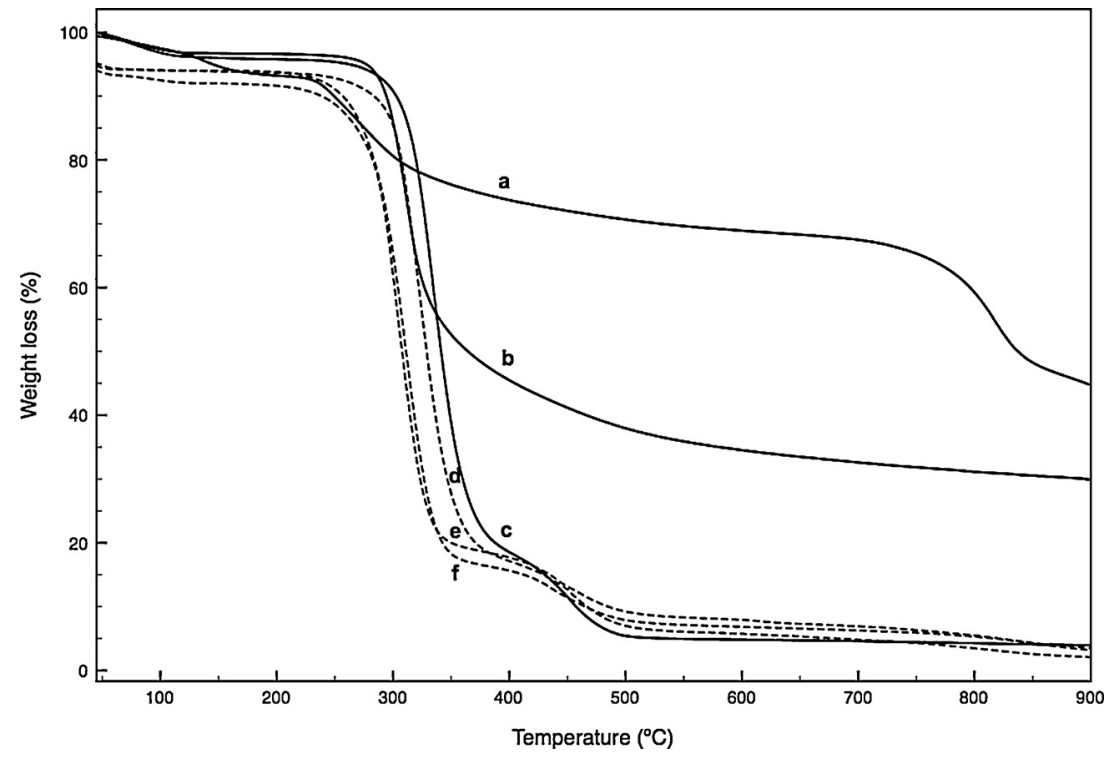

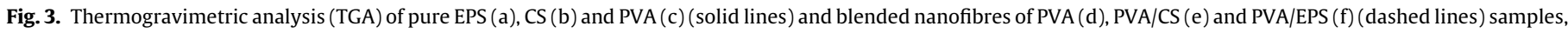
from $35^{\circ} \mathrm{C}$ to $900^{\circ} \mathrm{C}$, performed at a heating rate of $10^{\circ} \mathrm{C} / \mathrm{min}$, in a nitrogen atmosphere. 


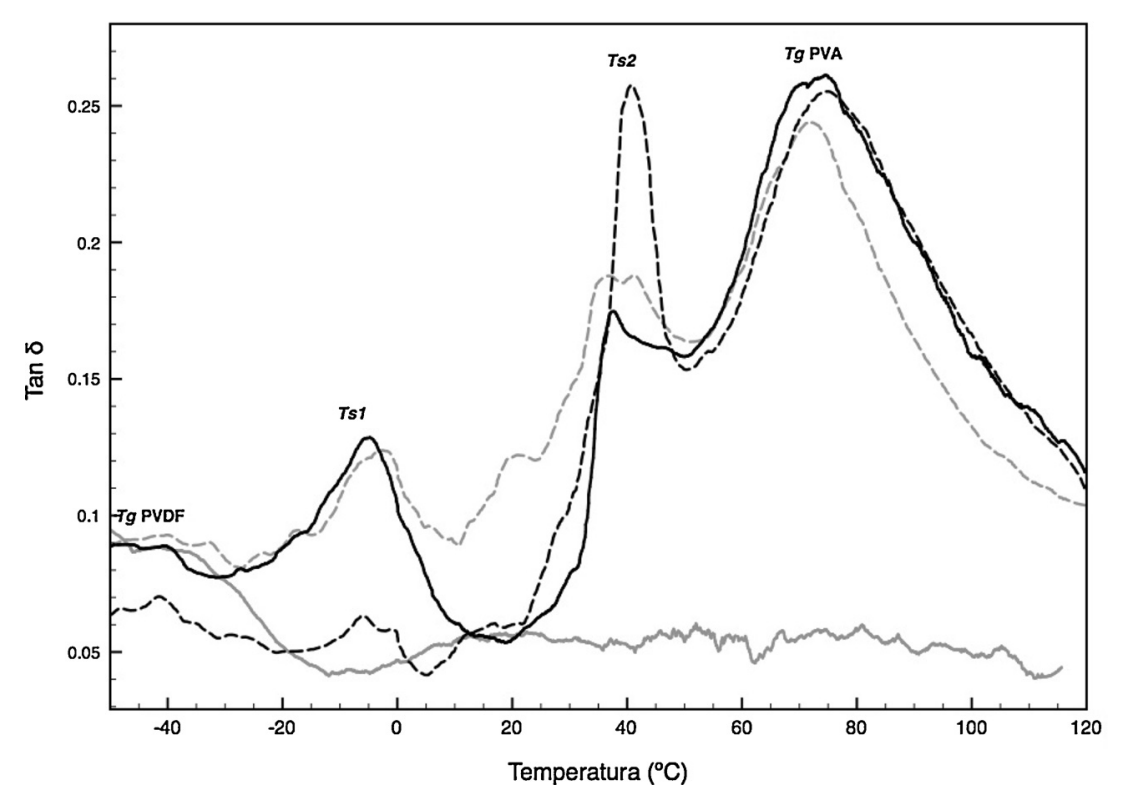

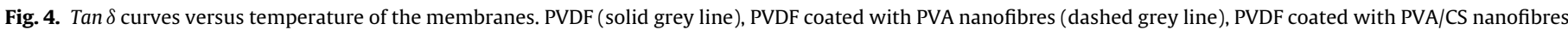
(dashed black line), and PVDF coated with PVA/EPS nanofibres (solid black line).

\subsection{Differential scanning calorimetry (DSC)}

The differential scanning calorimetry (DSC) analysis of PVA polymer revealed the presence of a glass transition temperature $\left(T_{g}\right)$ with a value of $70 \pm 2{ }^{\circ} \mathrm{C}$. The $T_{g}$ is lower than the usual value for highly hydrolysed PVA as a consequence of the relatively lower hydroxyl group content of this specific product, with a degree of hydrolysis between $87 \%$ and $90 \%$ (Krumova, Lopez, Benavente, Mijangos, \& Perena, 2000). EPS and chitosan showed an amorphous behaviour with no observable crystallization or fusion peaks in the measured range from $-50^{\circ} \mathrm{C}$ at up to $200^{\circ} \mathrm{C}$, with a rate of $10^{\circ} \mathrm{C} / \mathrm{min}$. Moreover, the DSC curve of the polysaccharides (not shown) does not show any readable $T_{g}$ features. Despite some crystalline regions exist, this behaviour is frequently detected in other semicrystalline polymers such as cellulose, due to their rigidrod polymer backbone having strong inter- and/or intra-molecular hydrogen bonding and to the rigid amorphous phase because of its heterocyclic units (Lee, Kim, \& Lee, 2000). As a result, the variations in heat capacity corresponding to the change in specific volume near $T_{g}$ are probably too small to be detected by the DSC technique. The DSC thermograms for PVA/CS and PVA/EPS polymer blend nanofibres show the same $T_{g}$ of the PVA nanofibres but with a slight positive shift in temperature. Usually, in a completely miscible blend of two polymers, a new single $T_{g}$ is observed in DSC thermograms between the $T_{g}$ values of each pure polymer. But, if two components are only partially miscible, the resulting polymer blend would exhibit two $T_{g}$ 's related to each component, but the $T_{g}$ value of each component phase could be affected by the other one (with a shift in glass transition temperature) and it is usually composition dependent (Suyatma, Copinet, Tighzert, \& Coma, 2004). Due to the fact that in the DSC analysis it was not possible to depict the $T_{g}$ values for the polysaccharides, difficulties can be encountered in the DSC interpretation if strong hydrogen-bonding interaction between polymers exists. A more sensitive method, the DMA technique, was therefore employed to further investigate the miscibility of the membranes.

\subsection{Dynamic mechanical analysis (DMA)}

Although the calorimetric and FTIR measurements suggested some level of miscibility between polysaccharide polymers and
PVA, further studies were needed to support this hypothesis. As far as the relaxation behaviour is concerned, DMA is a much more sensitive technique than DSC. The loss or damping factor $(\tan \delta)$ is the ratio of the loss modulus to the storage modulus and provides information on the relative contributions of the viscous and elastic components of a viscoelastic material. A high $\tan \delta$ value is indicative of a material that has a high, non-elastic strain component, while a low value indicates one that is more elastic. The DMA analysis was performed on the nanofibres mats electrospinned onto a polyvinylidene difluoride (PVDF) basal disc filter of $5 \mathrm{~cm}$ in diameter with a porosity of $0.2 \mu \mathrm{m}$ (Sterlitech, USA). As shown in Fig. 4, the variation of damping factor ( $\tan \delta$ ) versus temperature is highly affected by the inclusion of polysaccharides in the PVA formulation. The electrospinned membranes exhibit four regions with different elastic behaviour, represented by two secondary and two main relaxation temperatures. The two main $\alpha$-relaxation peaks at about $-42^{\circ} \mathrm{C}$ and $72^{\circ} \mathrm{C}$ are related to the glass transition temperatures of the PVDF membrane and to the PVA polymer, respectively, as previously reported (Cassu \& Felisberti, 1999; Gasmi, Gouasmia, \& Etienne, 2006). The broad and relatively high temperature peak between $-10^{\circ} \mathrm{C}$ and $-5^{\circ} \mathrm{C}\left(T_{s 1}\right)$ is attributed to the secondary $\beta$ relaxation temperature of PVA and polysaccharides in dry state. As a general trend, when PVA, cellulose, chitosan and other polysaccharides are dried, the $\beta$-relaxation shift towards higher temperatures (De La Rosa, Heux, \& Cavaille, 2000). The secondary relaxation temperature peaks between $35^{\circ} \mathrm{C}$ and $45^{\circ} \mathrm{C}\left(T_{s 2}\right)$ are associated with the local molecular motions or conformational changes of the PVA side groups (Cassu \& Felisberti, 1999). The polysaccharide blends showed different peak positions in this region when compared with PVA, suggesting that despite the low amount of CS and EPS in the blend, conformational changes in the PVA side groups happen and could be attributed to hydrogen bond assemblies (Quijada-Garrido, Iglesias-Gonzalez, Mazon-Arechederra, \& Barrales-Rienda, 2007). Moreover, the strong and sharp peak at about $45^{\circ} \mathrm{C}$ in PVA/CS blend membrane, could be attributed to an increase of residual water mobility in chitosan (Mucha \& Pawlak, 2005). Usually, blends of immiscible polymers exhibit identical glass transition temperatures to the unblended components. In the intermediate cases of partial polymer miscibility or if the size of the dispersed phase is very small, the $T_{g}$ of individual components may be shifted (Feldstein, Roos, Chevallier, Creton, \& Dormidontova, 2003). In 
our experiments no $T_{g}$ for chitosan or EPS could be retrieved and despite several works attempted to investigate the glass-transition phenomena in semi-crystalline polysaccharides, such as chitosan, the results are far from being in agreement. However, from the literature we may conclude that the glass transition of chitosan and cyanobacterial EPS should exist at temperatures above $90^{\circ} \mathrm{C}$, but this information is far from being straightforward (Lan, Wu, Zhang, Hu, \& Liu, 2010; Mano, 2008). Starting from this assumption, the slight enhancement in thermal stability, evidenced by the shifting towards higher values of temperature of the $T_{g}$ peaks of the blended PVA, could be attributed to intermolecular interactions between PVA and the polysaccharides due to hydrogen bonding, indicating that the system is very close to being miscible (Lee, Kim, \& Kim, 1996). The incorporation of the polysaccharide polymers seems to be primarily responsible for the decrease of $\tan \delta$ values in the temperature range between 10 and $30^{\circ} \mathrm{C}$ for the PVA/CS and PVA/EPS composite matrixes compared to the PVA matrix. However, only PVA/EPS composite has shown better mechanical properties up to $50^{\circ} \mathrm{C}$. These differences are considered to be the contribution of the well-organized double-network structure of the composite matrices, where the thermoplastic PVA networks were reinforced by the non-thermoplastic polysaccharide networks that hinders the chain mobility of the PVA polymer, resulting in the reduction of $\tan \delta$ value (Liu et al., 2004). The reduction in $\tan \delta$ also denotes an improvement in the hysteresis of the system and a reduction in the internal friction (Huda, Mohanty, Drzal, Schut, \& Misra, 2005). The enhancing of the elastic properties of the membrane in this temperature range is very important for the membrane performance in water filtration applications.

\subsection{Metal chelation test of electrospinned membranes}

Before the filtration step, the electrospun blend membranes assembled in the PVDF microfilter were cross-linked with glutaraldehyde to maintain its morphology and prevent it from dissolution during filtration. With the soft cross-linking conditions used, only a slight shrinkage of the membrane ( $\sim 5 \%)$ was found, and no significant changes in the nanofibres diameter, spatial distribution and thermo-mechanical properties were observed (data not shown).

The results of the hexavalent chromium dead-end ultrafiltration test in a high-pressure cell have shown a statistical-significant increase in chromium binding capacity of about $5 \%$ in both the polysaccharide blended membranes. This means that the membranes polysaccharides coating has retained $0.01 \mathrm{mg}$ per area $\left(\mathrm{m}^{2}\right)$, of $0.2 \mathrm{mg}$ of chromium, which pass through the membranes during the filtration process. This is a remarkable result given the low amount $(0.5 \%)$ of added polysaccharides.

The sorption mechanism on polysaccharides mainly depends on the structural properties of the polymer such as the number of monomer units, crystallinity, degree of branching and deacetylation. Moreover, the speciation, charge density, and electronic structure of the used metal ions are other important parameters that control sorption mechanism. Under the used neutral slightly basic $\mathrm{pH}$ filtration conditions, the major mechanism of metal uptake seems regulated by coordination to amine groups both present in chitosan and in the protein fraction of EPS and, in a lower extent, to the contribution of the hydroxyl groups. However, the significant results obtained with such a low amount of added polysaccharides in the nanofibres structure can also be explained by the Pearson's Hard Soft Acid Base (HSAB) theory, wherein the biosorbent acts as a Lewis base and the metal acts as a Lewis acid. According to this theory, metals ions are classified as hard, soft or borderline acids, depending to their affinity for different ligands. Smaller ions with higher charge densities, such as chromium, tend to behave as hard acids and bind preferentially to ligands of smaller size, such as the amine group on the blend nanofibers backbone, that behave as hard bases (Horzum, Boyaci, Eroglu, Shahwan, \& Demir, 2010).

\section{Conclusion}

Electrospun PVA/CS and PVA/EPS blend nanofibers have been successfully prepared. Blend nanofibres with uniform and smooth morphology with a narrow diameter distribution from about 50 to $130 \mathrm{~nm}$ were obtained and intermolecular hydrogen bonds between the biopolysaccharides and PVA were confirmed. DMA and filtration test demonstrated that the PVA/EPS electrospunblended membrane has better tensile mechanical properties and resisted more against disintegration in the temperature range between 10 and $50^{\circ} \mathrm{C}$. The metal chelation, thermal and mechanical properties of nanofibrous membranes developed using cyanobacterial polysaccharides show great potential for filtration purposes. This is the first successful report of electrospinned cyanobacterial polysaccharide.

\section{Acknowledgements}

This work was funded by FEDER funds through the Operational Competitiveness Programme - COMPETE and by National Funds through FCT - Fundação para a Ciência e a Tecnologia under the projects FCOMP-01-0124-FEDER-022718 (PEst-C/SAU/LA0002/2011), $\quad$ FCOMP-01-0124-FEDER-009389 (PTDC/CTM/100627/2008) and FCOMP-01-0124-FEDER-009697 (PTDC/EBB-EBI/099662/2008), and Grants SFRH/BPD/37045/2007 and SFRH/BPD/72400/2010.

\section{References}

Bhardwaj, N., \& Kundu, S. C. (2010). Electrospinning: A fascinating fiber fabrication technique. Biotechnology Advances, 28(3), 325-347.

Bonino, C. A., Krebs, M. D., Saquing, C. D., Jeong, S. I., Shearer, K. L., Alsberg, E., et al. (2011). Electrospinning alginate-based nanofibers: From blends to crosslinked low molecular weight alginate-only systems. Carbohydrate Polymers, 85(1), $111-119$

Cassu, S. N., \& Felisberti, M. I. (1999). Poly(vinyl alcohol) and poly(vinylpyrrolidone) blends: 2. Study of relaxations by dynamic mechanical analysis. Polymer, 40(17), 4845-4851.

Charernsriwilaiwat, N., Opanasopit, P., Rojanarata, T., Ngawhirunpat, T., \& Supaphol, P. (2010). Preparation and characterization of chitosanhydroxybenzotriazole/polyvinyl alcohol blend nanofibers by the electrospinning technique. Carbohydrate Polymers, 81(3), 675-680.

Chen, C. H., Wang, F. Y., Mao, C. F., Liao, W. T., \& Hsieh, C. D. (2008). Studies of chitosan: II. Preparation and characterization of chitosan/poly(vinyl alcohol)/gelatin ternary blend films. International Journal of Biological Macromolecules, 43(1), 37-42.

De La Rosa, A., Heux, L., \& Cavaille, J. Y. (2000). Secondary relaxations in poly(allylalcohol), PAA, and poly(vinyl alcohol), PVA Part I. Mechanical relaxations compared with mechanical behavior of cellulose and dextran in the presence of polar solvent. Polymer, 41(20), 7547-7557

De Philippis, R., Colica, G., \& Micheletti, E. (2011). Exopolysaccharide-producing cyanobacteria in heavy metal removal from water: molecular basis and practical applicability of the biosorption process. Applied Microbiology and Biotechnology, 92(4), 697-708.

Desai, K., Kit, K., Li, J., Michael Davidson, P., Zivanovic, S., \& Meyer, H. (2009). Nanofibrous chitosan non-wovens for filtration applications. Polymer, 50(15), 3661-3669

Duan, B., Yuan, X. Y., Zhu, Y., Zhang, Y. Y., Li, X. L., Zhang, Y., et al. (2006). A nanofibrous composite membrane of PLGA-chitosan/PVA prepared by electrospinning. European Polymer Journal, 42(9), 2013-2022.

Feldstein, M. M., Roos, A., Chevallier, C., Creton, C., \& Dormidontova, E. E. (2003) Relation of glass transition temperature to the hydrogen bonding degree and energy in poly(N-vinyl pyrrolidone) blends with hydroxyl-containing plasticizers: 3, Analysis of two glass transition temperatures featured for PVP solutions in liquid poly(ethylene glycol). Polymer, 44(6), 1819-1834.

Gasmi, A., Gouasmia, M., \& Etienne, S. (2006). Mechanical relaxations and transitions in poly(vinylidene fluoride) PVDF. Solid State Phenomena, 115, 151-156.

Gunn, J., \& Zhang, M. Q. (2010). Polyblend nanofibers for biomedical applications: Perspectives and challenges. Trends in Biotechnology, 28(4), 189-197.

Heikkila, P., \& Harlin, A. (2009). Electrospinning of polyacrylonitrile (PAN) solution: Effect of conductive additive and filler on the process. Express Polymer Letters, 3(7), 437-445. 
Horzum, N., Boyaci, E., Eroglu, A. E., Shahwan, T., \& Demir, M. M. (2010). Sorption efficiency of chitosan nanofibers toward metal ions at low concentrations. Biomacromolecules, 11(12), 3301-3308.

Huda, M. S., Mohanty, A. K., Drzal, L. T., Schut, E., \& Misra, M. (2005). Green composites from recycled cellulose and poly(lactic acid): Physico-mechanical and morphological properties evaluation. Journal of Materials Science, 40(16), 4221-4229.

Jia, Y.-T., Gong, J., Gu, X.-H., Kim, H.-Y., Dong, J., \& Shen, X.-Y. (2007). Fabrication and characterization of poly (vinyl alcohol)/chitosan blend nanofibers produced by electrospinning method. Carbohydrate Polymers, 67(3), 403-409.

Jia, Z. D., Li, Q., Liu, J. N., Yang, Y., Wang, L. M., \& Guan, Z. C. (2008). Preparation and properties of poly (vinyl alcohol) nanofibers by electrospinning. Journal of Polymer Engineering, 28(1/2), 87-100.

Kanekiyo, K., Hayashi, K., Takenaka, H., Lee, J. B., \& Hayashi, T. (2007). Anti-herpes simplex virus target of an acidic polysaccharide, nostoflan, from the edible blue-green alga Nostoc flagelliforme. Biological \& Pharmaceutical Bulletin, 30(8), 1573-1575

Krumova, M., Lopez, D., Benavente, R., Mijangos, C., \& Perena, J. M. (2000). Effect of crosslinking on the mechanical and thermal properties of poly(vinyl alcohol). Polymer, 41(26), 9265-9272.

Kumar, C. G., Joo, H.-S., Choi, J.-W., Koo, Y.-M., \& Chang, C.-S. (2004). Purification and characterization of an extracellular polysaccharide from haloalkalophilic Bacillus sp I-450. Enzyme and Microbial Technology, 34(7), 673-681.

Lan, S. B., Wu, L., Zhang, D. L., Hu, C. X., \& Liu, Y. D. (2010). Effects of drought and salt stresses on man-made cyanobacterial crusts. European Journal of Soil Biology, 46(6), 381-386

Lau, W. J., Ismail, A. F., Misdan, N., \& Kassim, M. A. (2012). A recent progress in thin film composite membrane: A review. Desalination, 287, 190-199.

Lee, S. J., Kim, S. S., \& Lee, Y. M. (2000). Interpenetrating polymer network hydrogels based on poly(ethylene glycol) macromer and chitosan. Carbohydrate Polymers, 41(2), 197-205.

Lee, Y. M., Kim, S. H., \& Kim, S. J. (1996). Preparation and characteristics of beta-chitin and poly(vinyl alcohol) blend. Polymer, 37(26), 5897-5905

Leung, V., \& Ko, F. (2011). Biomedical applications of nanofibers. Polymers for Advanced Technologies, 22(3), 350-365.

Liu, L. S., Won, Y. J., Cooke, P. H., Coffin, D. R., Fishman, M. L., Hicks, K. B., et al. (2004). Pectin/poly(lactide-co-glycolide) composite matrices for biomedical applications. Biomaterials, 25(16), 3201-3210.

Liu, Y., Wang, R., Ma, H. Y., Hsiao, B. S., \& Chu, B. (2013). High-flux microfiltration filters based on electrospun polyvinylalcohol nanofibrous membranes. Polymer, 54(2), 548-556.

Mano, J. F. (2008). Viscoelastic properties of chitosan with different hydration degrees as studied by dynamic mechanical analysis. Macromolecular Bioscience, 8(1), 69-76.

Mansur, H. S., Sadahira, C. M., Souza, A. N., \& Mansur, A. A. P. (2008). FTIR spectroscopy characterization of poly (vinyl alcohol) hydrogel with different hydrolysis degree and chemically crosslinked with glutaraldehyde. Materials Science \& Engineering C: Biomimetic and Supramolecular Systems, 28(4), 539-548.
Mbhele, Z. H., Salemane, M. G., van Sittert, C. G. C. E., Nedeljkovic, J. M., Djokovic, V. \& Luyt, A. S. (2003). Fabrication and characterization of silver-polyvinyl alcohol nanocomposites. Chemistry of Materials, 15(26), 5019-5024.

Mota, R., Guimarães, R., Büttel, Z., Rossi, F., Colica, G., Silva, C. J., et al. (2013) Production and characterization of extracellular carbohydrate polymer from Cyanothece sp, CCY 0110. Carbohydrate Polymers, 92(2), 1408-1415.

Mucha, M., \& Pawlak, A. (2005). Thermal analysis of chitosan and its blends. Thermochimica Acta, 427(1/2), 69-76.

Peng, Z., \& Kong, L. X. (2007). A thermal degradation mechanism of polyviny alcohol/silica nanocomposites. Polymer Degradation and Stability, 92(6), 1061-1071.

Pereira, S., Micheletti, E., Zille, A., Santos, A., Moradas-Ferreira, P., Tamagnini, P., et al. (2011). Using extracellular polymeric substances (EPS)-producing cyanobacteria for the bioremediation of heavy metals: Do cations compete for the EPS functional groups and also accumulate inside the cell? Microbiology-Sgm, 157, 451-458.

Pereira, S., Zille, A., Micheletti, E., Moradas-Ferreira, P., De Philippis, R., \& Tamagnini, P.(2009). Complexity of cyanobacterial exopolysaccharides: composition, structures, inducing factors and putative genes involved in their biosynthesis and assembly. FEMS Microbiology Reviews, 33(5), 917-941.

Peresin, M. S., Habibi, Y., Zoppe, J. O., Pawlak, J. J., \& Rojas, O. J. (2010). Nanofiber composites of polyvinyl alcohol and cellulose nanocrystals: Manufacture and characterization. Biomacromolecules, 11(3), 674-681.

Quijada-Garrido, I., Iglesias-Gonzalez, V., Mazon-Arechederra, J. M., \& BarralesRienda, J. M. (2007). The role played by the interactions of small molecules with chitosan and their transition temperatures, Glass-forming liquids: 1,2,3propantriol (glycerol). Carbohydrate Polymers, 68(1), 173-186.

Schiffman, J. D., \& Schauer, C. L. (2008). A review: Electrospinning of biopolymer nanofibers and their applications. Polymer Reviews, 48(2), 317-352.

Shao, C. L., Kim, H. Y., Gong, J., Ding, B., Lee, D. R., \& Park, S. J. (2003). Fiber mats of poly(vinyl alcohol)/silica composite via electrospinning. Materials Letters, 57(9/10), 1579-1584.

Suyatma, N. E., Copinet, A., Tighzert, L., \& Coma, V. (2004). Mechanical and barrier properties of biodegradable films made from chitosan and poly (lactic acid) blends. Journal of Polymers and the Environment, 12(1), 1-6.

Tang, Z., Qiu, C., McCutcheon, J. R., Yoon, K., Ma, H., Fang, D., et al. (2009). Design and fabrication of electrospun polyethersulfone nanofibrous scaffold for highflux nanofiltration membranes. Journal of Polymer Science Part B: Polymer Physics, 47(22), 2288-2300.

Wang, X. F., Chen, X. M., Yoon, K., Fang, D. F., Hsiao, B. S., \& Chu, B. (2005). High flux filtration medium based on nanofibrous substrate with hydrophilic nanocomposite coating. Environmental Science E Technology, 39(19), 7684-7691.

Yan, E., Fan, S., Li, X., Wang, C., Sun, Z., Ni, L., et al. (2013). Electrospun polyvinyl alcohol/chitosan composite nanofibers involving Au nanoparticles and their in vitro release properties. Materials Science and Engineering: C, 33(1), 461-465. 